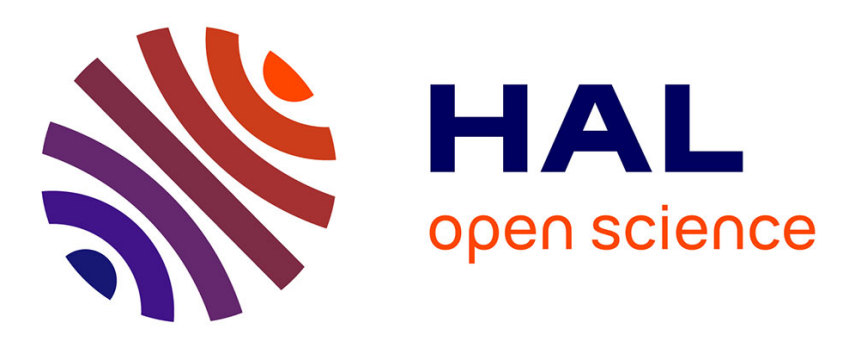

\title{
Reverberation Chamber Modeling Based on Image Theory: Investigation in the Pulse Regime
}

\author{
Emmanuel Amador, Christophe Lemoine, Philippe Besnier, Alexandre Laisné
}

\section{To cite this version:}

Emmanuel Amador, Christophe Lemoine, Philippe Besnier, Alexandre Laisné. Reverberation Chamber Modeling Based on Image Theory: Investigation in the Pulse Regime. IEEE Transactions on Electromagnetic Compatibility, 2010, 52 (4), pp.778-789. 10.1109/TEMC.2010.2049576 . hal-00537133

\section{HAL Id: hal-00537133 \\ https://hal.science/hal-00537133}

Submitted on 17 Nov 2010

HAL is a multi-disciplinary open access archive for the deposit and dissemination of scientific research documents, whether they are published or not. The documents may come from teaching and research institutions in France or abroad, or from public or private research centers.
L'archive ouverte pluridisciplinaire HAL, est destinée au dépôt et à la diffusion de documents scientifiques de niveau recherche, publiés ou non, émanant des établissements d'enseignement et de recherche français ou étrangers, des laboratoires publics ou privés. 


\title{
Reverberation Chamber Modeling Based on Image Theory: Investigation in the Pulse Regime
}

\author{
Emmanuel Amador, Student, IEEE, Christophe Lemoine, Philippe Besnier, Member, IEEE,
} and Alexandre Laisné

\begin{abstract}
In this paper we propose a straightforward $3 \mathrm{D}$ time domain model of a reverberation chamber (RC) based on image theory. This model allows one to describe the earliest moments of an arbitrary waveform in an RC. Time domain and frequency domain results from this model are analyzed and compared with measurements conducted in a reverberation chamber.

Index Terms - frequency domain, image theory, lowest usable frequency, model, pulse regime, reverberation chamber, time domain analysis, transients.
\end{abstract}

\section{INTRODUCTION}

\section{A. Time Domain Reverberation Chamber Modeling}

Reverberation chambers $(\mathrm{RC})$ are traditionally used and studied in frequency domain to conduct electromagnetic compatibility (EMC) measurements either for electromagnetic immunity (EMI) or electromagnetic susceptibility (EMS) assessments. Their study in the time domain and in the pulse regime becomes relevant with regard to high intensity radiated field testing. In such immunity tests to radar-like signals, the incident waveform may be strongly altered by the intrinsic nature of the reverberation chamber (Fig. 1). Therefore a comprehensive view of an $\mathrm{RC}$ in the time domain through modeling would be advantageous to study the transients and the effect of loading on the functioning of the chamber to name some essential questions about the pulse regime in an RC.

Preliminary experimental investigations in [1], [2] with a real radar source deliver some insights into the effect of loading on the levels obtained for EMI testing. Transients in an RC have also been investigated in [3] from the viewpoint of mode tuned operations.

E. Amador, C. Lemoine and P. Besnier are with the Institute of Electronics and Telecommunications of Rennes (IETR), INSA of Rennes, Rennes 35043, France, mail: emmanuel.amador@insa-rennes.fr,

A. Laisné is with the Centre d'Essais Aéronautique de Toulouse (CEAT) of the Délégation Générale de l'Armement (DGA) in Toulouse, 31131 Balma, France.

DRAFT VERSION, GO TO IEEEXPLORE FOR THE FINAL VERSION

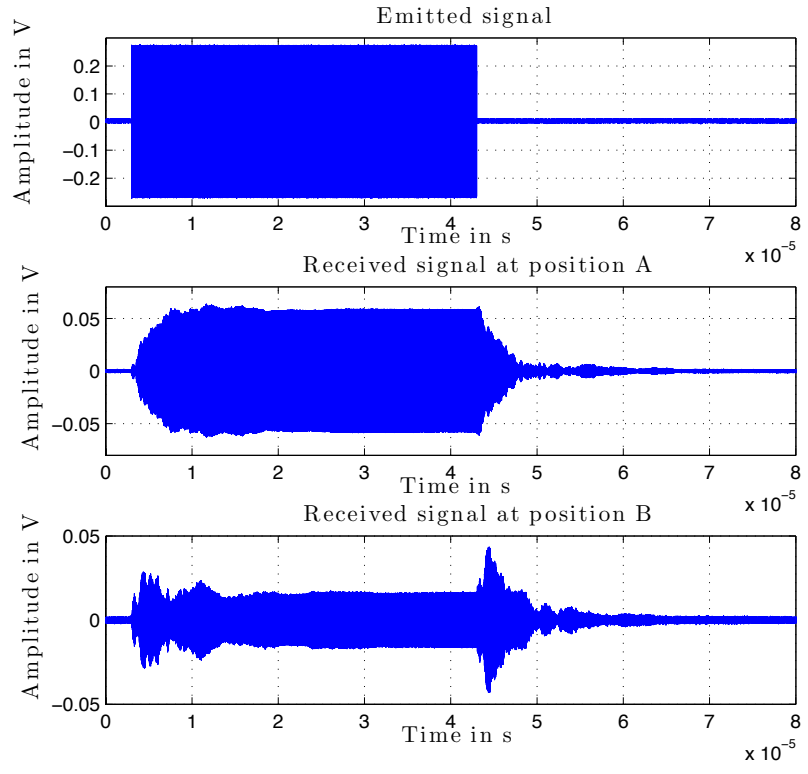

Fig. 1. Signal measured in a $\mathrm{RC}$ with a $40 \mu$ s sinusoidal pulse at $1 \mathrm{GHz}$ (above) at two different positions A (middle) and $\mathrm{B}$ (bottom) separated by $30 \mathrm{~cm}$ (no stirring process).

Very little modeling work is available in the literature to study transients. Use of transmission line matrix (TLM) or finite difference time domain (FDTD) simulations are possible solutions for simulating an RC [4]-[7], but using a space-time discretization of highly conductive and electrically large cavities induces severe limitations. Rather than using standard Maxwell equations, using their asymptotic approximations may reveal the intrinsic behavior of RC cavities. Optical techniques like ray tracing (RT) discretize the environment and are not able to simulate hundreds of reflections and so cannot reproduce the reverberation phenomenon in the chamber.

Image theory is a very straightforward approach that allows one to simulate hundreds of reflections and so to reproduce a reverberation environment. In [8], the authors use image theory to model a rectangular shielded cavity. An asymptotic approach for time domain simulations is used to 
extract the quality factor of the shielded cavity at a given frequency. To our knowledge it is the first paper about a time domain analysis of a $\mathrm{RC}$ based upon an optical model. In another paper [9], the authors investigate various approaches to model an RC. A 2D and a 3D model based on image theory are proposed. But the hardware limitations of the computer at the time did not allow them to simulate enough energy to converge for a frequency analysis of the simulated cavities. Capabilities of modern computers give a second impetus to optical approaches for RCs.

\section{B. Image Theory for a Reverberation Chamber}

Our model is based upon image theory [10]. In [10], a representation of a rectangular waveguide through image theory is given. Our model is an adaptation of this model of a waveguide to the geometry of a rectangular cavity. It consists in closing the waveguide by adding two boundary conditions. In our model, an elementary current is placed in the cavity. The positions and the angular orientations of image-current created by the reflective walls must be determined. This very straightforward model does not directly involve Maxwell's equations nor a spatial discretization of the environment. It fits the simple geometry of a shielded cavity. It only uses the far-field ${ }^{1}$ and free-space radiation approximation of millions of elementary currents to represent a complex interaction between an elementary current and electrical conductor boundaries.

The core of the simulation is an impulse response. By convolving the impulse response with a chosen waveform, the model is able to simulate the behavior of the $\mathrm{RC}$ with a particular waveform. By applying a Fourier transform on the impulse response, we can explore the frequency domain. The rough representation of the $\mathrm{RC}$ by a rectangular shielded cavity without a mode stirrer is too elementary to obtain deterministic results. But it has been shown that source stirring is equivalent to mechanical stirring [11]-[14] and so from a statistical point of view taking randomly $N$ receiver positions is equivalent to taking $N$ stirrer positions. In our model, the stirrer is not described but its absence will not affect the statistics of the results as long as the receiver positions are taken randomly.

This paper is dedicated to the presentation of our model and its use for two applications. After a detailed explanation of our model, preliminary elementary results are given to verify the physical consistency of our model. Two applications of our model are presented, an estimation of the lowest

\footnotetext{
${ }^{1}$ Near field radiation are not simulated but can be easily added to the model
}

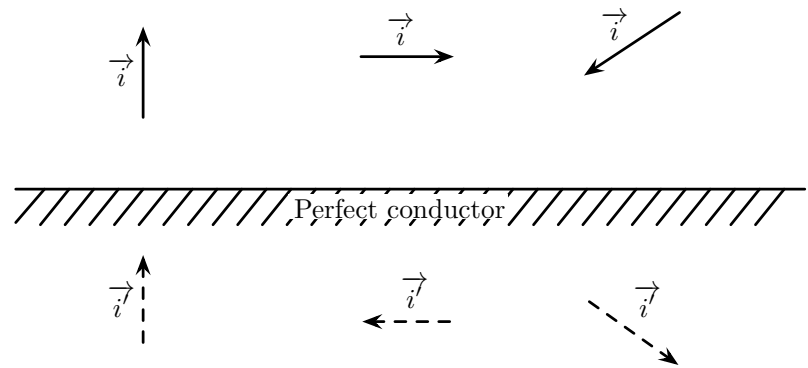

Fig. 2. Image theory applied to electrical currents.

usable frequency (LUF) and a statistical analysis of waveforms obtained in the pulse regime. The results obtained with this model are analyzed and compared statistically with measurements made in the RC in our laboratory at the Institute of Electronics and Telecommunications of Rennes (IETR).

\section{Shielded Cavity Model}

\section{A. Image Theory}

Image theory is generally introduced with electric charges. Let a negative charge be placed at a distance $d$ from an infinite perfectly conducting plane. This conductive plane is an anti-symmetrical plane, thus a positive charge is facing the negative charge. The resulting field of the negative charge and the plane is the field created by an electrostatic dipole with the two charges. Image theory can be applied to moving charges. Fig. 2 sums up the different possible configurations with an electric current vector $\vec{i}$. Fig. 3, inspired by [10], presents a vertical and an horizontal view of the image currents created by applying the construction rules presented in Fig. 2 to an arbitrarily oriented current in a rectangular cavity. The real cavity (in bold line, in the middle) is surrounded by image cavities. Each image cavity contains an image current. We define the order of an image current, i.e, the order of an image cavity, as the number of reflections involved in its creation. The number of cavities for a given order $n>0$, is given by

$$
N_{n}=4 n^{2}+2
$$

and the total number of cavities till the order $n$ is given by

$$
\begin{aligned}
M_{n} & =1+\sum_{i=1}^{n}\left(4 i^{2}+2\right) \\
& =1+2 n+\frac{2 n(n+1)(2 n+1)}{3} .
\end{aligned}
$$

The growth of $M_{n}$ is therefore proportional to $n^{3}$.

1) Generation of the image currents: Let a rectangular cavity be of length $l$, width $p$, and height $h$. A corner of this cavity is the origin of the rectangular coordinates and the three main directions 


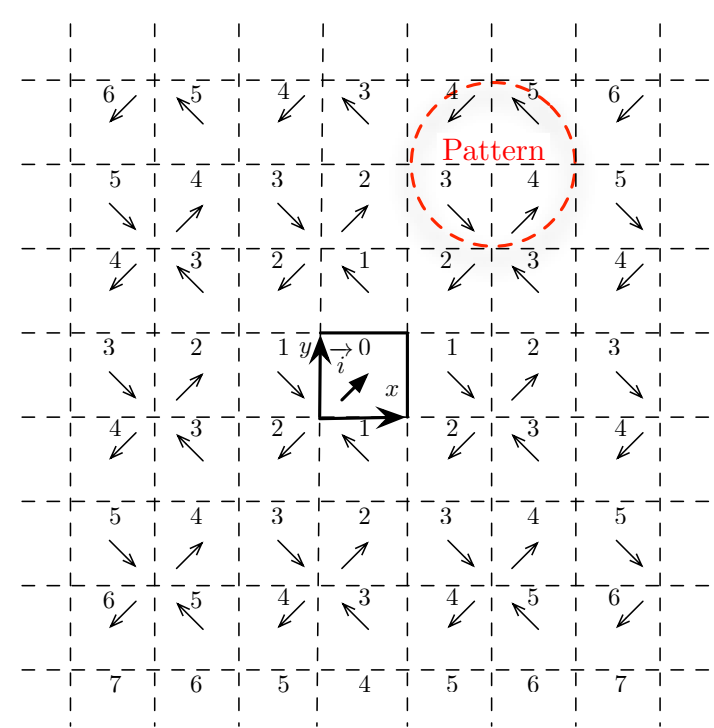

(a)

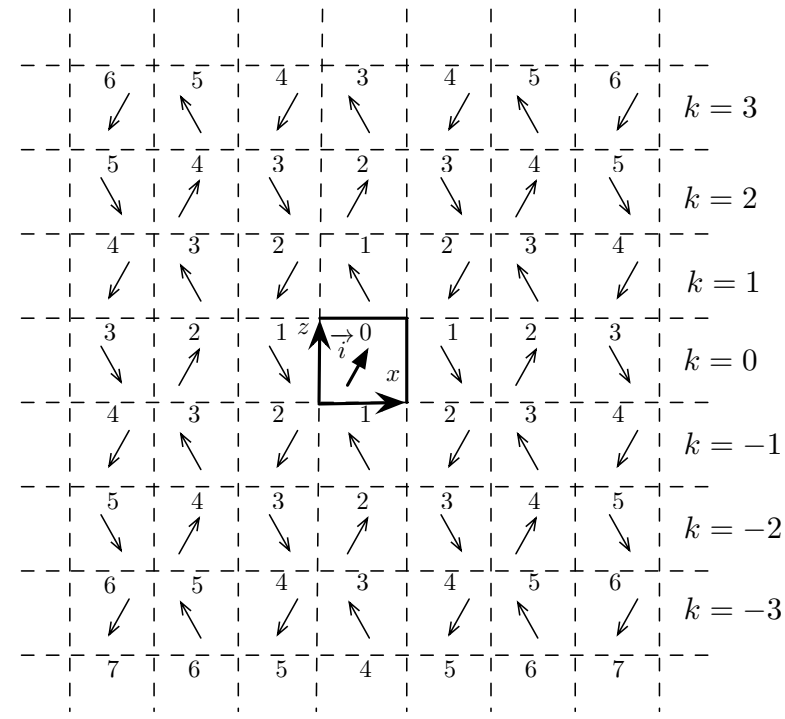

(b)

Fig. 3. Image cavities and image currents in an horizontal plane $(k=0)(\mathrm{a})$ and a vertical plane $(j=0)(\mathrm{b})$. The order of each cavity is indicated.

$O x, O y$, and $O z$ are defined by the edges of the cavity.

Let an elementary current be placed within this cavity at the point $A\left(x_{0}, y_{0}, z_{0}\right)$, its angular orientation in the cavity is defined by a tilt angle $\alpha$ and an azimuthal angle $\beta$ as presented in Fig. 4 . Generating the elementary image current means that we have to determine the position and the angular orientation of every current created by the reflections with the walls and save the number of reflections involved for each direction. A oneby-one image creation process can take days to generate millions of sources. The first step is to identify patterns in Fig. 3 to speed-up the image creation process. An elementary current can be identified by the reflections involved in its creation. If we consider an $n^{\text {th }}$ order current created by $i$ reflections along the axis $O x, j$ reflections along the axis $O y, k$ reflections along the axis $O z$, we have $n=|i|+|j|+|k|^{2}$.

If we examine the horizontal plane $(k=0)$ represented in Fig. 3(a), we can identify a pattern of four juxtaposed elementary currents (Fig. 3(a)). This pattern is duplicated in the whole horizontal plane to create the plane $k=0$ of elementary currents.

If we study the orientations of the elementary currents in a vertical plane (Fig. 3(b)), we can discern that the parity of $k$ dictates the orientation of the current in the corresponding horizontal plane. There are only two different horizontal planes. We

\footnotetext{
${ }^{2}$ The numbers $i, j$ and $k$ can be negative if the reflections are in the decreasing direction along the respective axis.
}

already have the plane $k=0$ and thus all the even horizontal planes. We can easily derive the odd planes from an even plane, the positions of the currents along the axis $O x$ and $O y$ are the same and the vertical positions of the elementary currents differ. The height of all the currents in the $k=0$ horizontal plane is $z_{0}$ and the height of all the currents in the horizontal plane $k=1$ is $2 l-z_{0}$. The tilt angles are conserved but the azimuthal angles of the currents are reversed. We add $\pi$ radians to the azimuthal angle of the currents of the plane $k=0$ to obtain the azimuthal angles of the currents contained in an odd plane. These two horizontal plane configurations are then duplicated along the $\mathrm{O} z$ direction, the vertical positions of the generated sources and the number of reflections along the axis $O z$ are adjusted accordingly.

2) Cavity loss: Image theory is applied with perfectly conducting materials. To simulate a lossy rectangular cavity, we introduce three loss coefficients $R_{x}, R_{y}, R_{z}$ corresponding to the three main directions of propagation. ${ }^{3}$ Unlike in [8] where complex reflection coefficients are used because the losses in the cavity are a function of the conductivity of the walls, the loss coefficients in our model include the losses created by one reflection on a wall and by the lossy objects in the cavity for a ray traveling along a given direction. These loss coefficients are average loss coefficient as if all the losses in the cavity were from an absorbing

\footnotetext{
${ }^{3}$ In this paper, the three coefficients have the same value $R$. In some situations it can be helpful to have three distinct coefficients to simulate an open door or a lossy wall.
} 


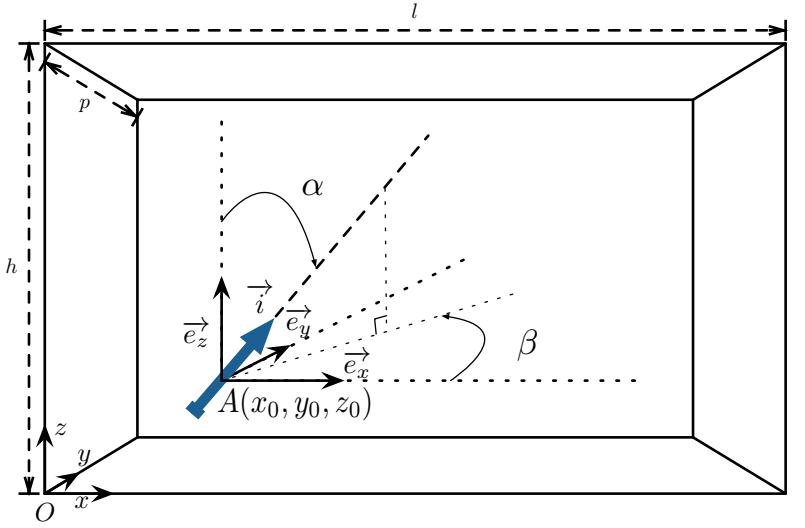

Fig. 4. Angles and coordinates of the elementary dipole in the cavity.

paint spread on the cavity walls. In this section we focus on an elementary current $a$ inside a $n^{\text {th }}$ order image-cavity. This elementary current is created by $i$ reflections along the $O x$ axis, $j$ reflections along the $O y$ axis, and $k$ reflections along the $O z$ axis. The attenuation associated to this elementary current $a$ is:

$$
R_{a}=R_{x}^{|i|} R_{y}^{|j|} R_{z}^{|k|}, \text { with }|i|+|j|+|k|=n .
$$

The magnitude of this current can be written:

$$
I_{a}=I_{0} \cdot R_{a},
$$

where $I_{0}$ is the intensity of every current in the system if the walls are perfectly conducting. Assuming $R=R_{x}=R_{y}=R_{z}$, and if we neglect the cross product, the maximal amount of energy possible $\mathcal{E}_{\text {tot }}$ found in the system is proportional to:

$$
\mathcal{E}_{\text {tot }} \propto I_{0}^{2}+\sum_{i=1}^{\infty}\left(4 i^{2}+2\right) I_{0}^{2} \cdot R^{2 i} .
$$

As $R<1$, the sum above converges and the system described by the model is stable.

3) Channel impulse response simulation: The intensity of the particular elementary current $a, \overrightarrow{I_{a}}$ can be written:

$$
\overrightarrow{I_{a}}=I_{a} \cdot \vec{w}=I_{0} R_{x}^{|i|} R_{y}^{|j|} R_{z}^{|k|} \cdot \vec{w},
$$

where $\vec{w}$ is the normalized vector along the direction of the considered elementary current. The current in the real cavity and all the image currents simultaneously emit an elementary impulse $f(t)$. The intensity of the elementary current $a$ can be written:

$I_{a}(t)=I_{0} R_{a} \cdot f(t)$, with $f(t)= \begin{cases}1 & \text { if } t=0 \\ 0 & \text { otherwise }\end{cases}$

The orientation of the current at this position is given by a tilt angle $\alpha$, defined by the angle between $\vec{w}$ and $\overrightarrow{e_{z}}$ and an azimuthal angle $\beta$ defined by the angle between $\vec{w}-\left(\vec{w} \cdot \overrightarrow{e_{z}}\right) \vec{w}$ and $\overrightarrow{e_{x}}$. The electrical field created by the elementary current $a$ and received at a reception point $P$ within the real cavity can be written ${ }^{4}$

$\overrightarrow{E_{a}}(t)=-\omega \mu \frac{d h I_{0} R_{a} f\left(t-t_{a}\right)}{4 \pi d_{a}} \sin \theta_{a}\left\{\begin{array}{r}\cos \theta_{a} \cos \phi_{a} \cdot \vec{u} \\ \cos \theta_{a} \sin \phi_{a} \cdot \vec{v} \\ -\sin \theta_{a} \cdot \vec{w}\end{array}\right.$

with:

$$
\left\{\begin{array}{l}
\vec{u}=\mathcal{R}_{\alpha, \beta} \cdot \overrightarrow{e_{x}} \\
\vec{v}=\mathcal{R}_{\alpha, \beta} \cdot \overrightarrow{e_{y}} \\
\vec{w}=\mathcal{R}_{\alpha, \beta} \cdot \overrightarrow{e_{z}}
\end{array}\right.
$$

where $d h$ is the length of the elementary dipole, $d_{a}$ is the distance between the position of the elementary current $a$ and the reception point $P$, $t_{a}$ is the time of arrival at the reception point, and $\theta_{a}$ and $\phi_{a}$ are angular coordinates of the point $P$ in the local spherical coordinate system attached to the elementary current $a . \vec{u}, \vec{v}$, and $\vec{w}$ define the local rectangular basis attached to the elementary current. $\mathcal{R}_{\alpha, \beta}$ is the rotation matrix ${ }^{5}$ that changes the rectangular basis $\left(\overrightarrow{e_{x}}, \overrightarrow{e_{y}}, \overrightarrow{e_{z}}\right)$ into the local basis $(\vec{u}, \vec{v}, \vec{w})$ and $c$ is the celerity.

From the E-field expression (8) in the local rectangular coordinate system attached to the elementary current, we can deduce the expression in the rectangular coordinate system attached to the simulated cavity:

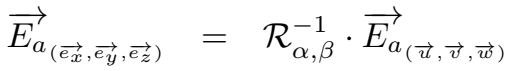

$$
\begin{aligned}
& =\mathcal{R}_{-\alpha, \beta} \cdot{\overrightarrow{E_{a(\vec{u}, \vec{v}, \vec{w})}}} .
\end{aligned}
$$

The channel impulse response (CIR) is given by adding the contribution of every current in our system. If $M$ is the total number of currents in our system, from (8) we can deduce three CIRs corresponding to the three rectangular components:

$$
s_{x, y, z}(t)=\sum_{i=0}^{M} \vec{E}_{i}(t) \cdot \overrightarrow{e_{x, y, z}} .
$$

The CIR can be convoluted with a chosen signal to simulate the waveform obtained at the position $P$ in the shielded cavity. By applying a Fourier transform on the CIR, the frequency domain of the cavity can be studied.

$4(8)$ is valid for a dipole radiation pattern $(-\sin \theta)$. One should note that any $3-\mathrm{D}$ radiation pattern can be employed.

${ }^{5} \mathcal{R}_{\alpha, \beta}$ represents a rotation of an angle $\alpha$ around a unitary vector $\vec{e}_{\beta}=-\cos \beta \overrightarrow{e_{x}}+\sin \beta \overrightarrow{e_{y}}$,

$\mathcal{R}_{\alpha, \beta}=\left(\begin{array}{ccc}\cos ^{2} \beta+\left(1-\cos ^{2} \beta\right) \cos \alpha & -\cos \beta \sin \beta(1-\cos \alpha) & \sin \beta \sin \alpha \\ -\cos \beta \sin \beta(1-\cos \alpha) & \sin ^{2} \beta+\left(1-\sin ^{2} \beta\right) \cos \alpha \cos \beta \sin \alpha \\ -\sin \beta \sin \alpha & -\cos \beta \sin \alpha & \cos \alpha\end{array}\right)$ 
One should note that the quantity $C=-\omega \mu \frac{d h I_{0}}{4 \pi}$ equals $1 \mathrm{~V}$ in our simulations. It means that the quantity $\omega d h$ is constant and independent of the frequency. This approximation is adequate if the power radiated by the antenna used for the measurements is constant for the considered bandwidth. As our model cannot pretend to be deterministic, obtaining absolute values is not necessary. The E-fields in this paper are expressed in $\mathrm{V} . \mathrm{m}^{-1}$ but the values are arbitrary. However, when the levels obtained by simulation are compared to measured levels, we introduce an invariant correction factor (or bias for values in $\mathrm{dB}$ ).

\section{B. Assumptions}

Using image theory to model a shielded cavity means that we omit the energy diffracted by the wall edges in the cavity. We only consider the energy reflected by the different walls. This optical approach is validated if the dimensions the cavity involved are substantially bigger than the wavelength $^{6}$. In these conditions the geometrical laws of optics can be applied to the image current emissions. This model uses far-field radiation only. The interaction between the emitting antenna and the device under test in a reverberation chamber implies more often far field radiation than near field radiation. Near-field radiations are neglected but they can easily be added to (8) to suit a particular configuration. The radiating current does not have any physical dimension, but this limitation can be easily bypassed by juxtaposing emitting currents to simulate a radiating line or an antenna in the cavity.

\section{Discussion: Applicability and Limitations}

1) Applicability: Our model was designed to measure the effects of a parameter on the waveforms obtained in the cavity. The parameters of our model that can be modified are:

- the dimensions of the cavity, our model is able to simulate any rectangular cavity,

- the waveform, our model is able to reproduce the behavior of the cavity for any arbitrary signal,

- the loading of the cavity, it can be simulated by precisely adjusting the loss coefficients,

- the directivity of the emitting source, it can be tuned by modifying the radiation of the elementary currents or by creating an array antenna with elementary currents.

\footnotetext{
${ }^{6}$ However, results presented in III-B show a good adequacy between measurements and simulations even at low frequency.
}

2) Limitations: The model presented in this paper is designed for an empty shielded cavity. To include a stirrer or an object in the cavity, the environment must be discretized and this straightforward model would become a complex ray-tracing model. Our approach here is to preserve the simplicity of the model. The presence of a lossy object inside the cavity can be integrated by reducing the loss coefficients. Because the exploitation of this model is statistical, the effect of this approximation on the time-domain response is reduced. The mechanical stirring process can be replaced by moving the emitter or the receiver (source stirring) [11][14]. In the frequency domain, however, electronic stirring provides an efficient stirring process, as long as the frequencies considered are independent [15]. Our model aims to represent roughly the behavior of an RC and the simulations presented in this paper will be analyzed statistically and compared to measurements conducted in a real RC.

If the model is relatively straightforward, the memory usage of the algorithm can be a problem. Image-currents are generated numerically and their positions and various attributes ${ }^{7}$ are stored in a matrix. The number of sources is given by (2). In reality the main parameter of the simulation is not the maximum order but the duration of the simulated time-window $L_{T}$. It means that we only need the image-currents within a radius $c \cdot L_{T}$. This filtering can save a lot of memory if the $\mathrm{RC}$ is not cubic.

Table I sums up the memory usage for a given time window. On a 64 bits platform with $32 \mathrm{~GB}$ of memory, we manage to reach a time-window of 3 $\mu \mathrm{s}$.

TABLE I

LENGTH OF THE SIMULATED TIME-WINDOWS AND MEMORY USAGE FOR THE RC AT THE IETR.

\begin{tabular}{c|c|c}
$L_{T}$ & Number of currents $M$ & Memory usage \\
\hline $500 \mathrm{~ns}$ & $1.1 \times 10^{6}$ & $90 \mathrm{MB}$ \\
$1 \mu \mathrm{s}$ & $8.8 \times 10^{6}$ & $700 \mathrm{MB}$ \\
$3 \mu \mathrm{s}$ & $238 \times 10^{6}$ & $19 \mathrm{~GB}$ \\
$10 \mu \mathrm{s}$ & $8.8 \times 10^{9}$ & $700 \mathrm{~GB}$ \\
$100 \mu \mathrm{s}$ & $8.8 \times 10^{12}$ & $700 \mathrm{~TB}$
\end{tabular}

Measurements in our RC at the IETR have shown that $99 \%$ of the energy of an impulse response is contained in $20 \mu$ s when the cavity is empty. This means that the model we propose can only simulate the first moments of our empty

\footnotetext{
${ }^{7}$ For each current the attributes are: the rectangular coordinates, the number of reflections along each axis, tilt and azimuth angles, the amplitude and the phase of the current for array antenna.
} 

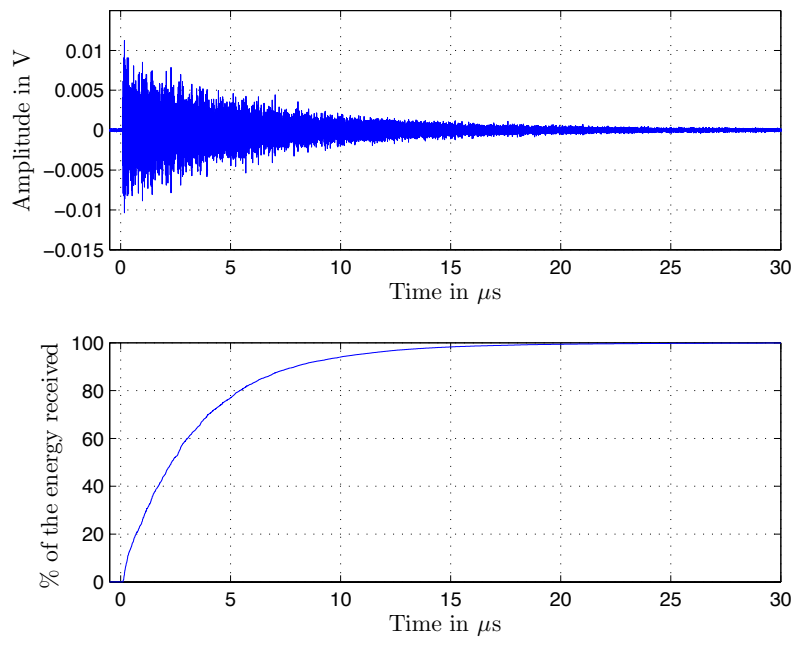

Fig. 5. Measured channel impulse response at a given reception point in the empty IETR RC (top) and percentage of the total energy of the impulse response vs. time (bottom).

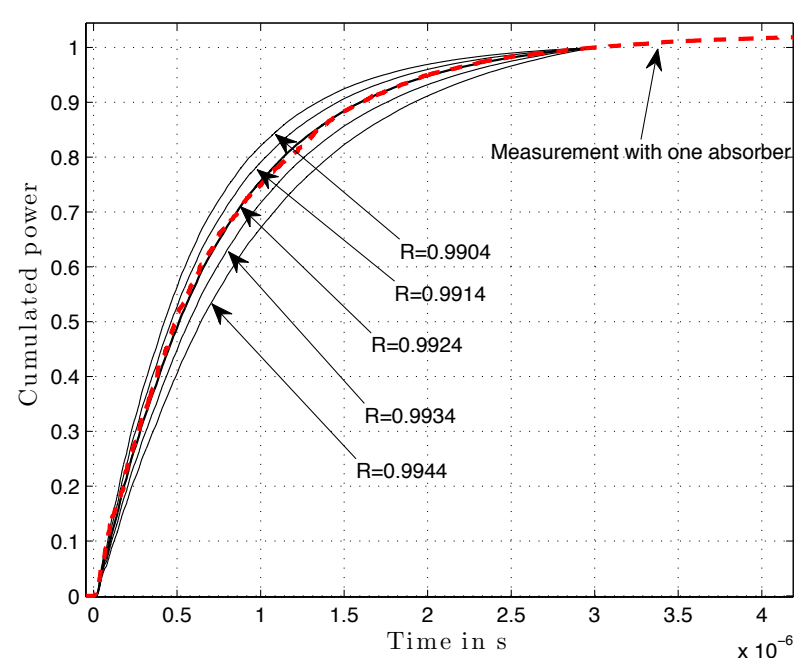

Fig. 6. Measured cumulated power with one absorber vs. time and simulations with different values of $R$. In this case the value $R=0.9924$ fits the measurement.

cavity. We can note that $60 \%$ of the energy is contained in the first $3 \mu \mathrm{s}$ (Fig. 5) when the RC is empty. Moreover this restriction is less problematic in the pulse regime and/or with a loaded cavity. If the length of the pulse is smaller than the timewindow, we can reasonably expect that the maximum levels would appear within the time-window. If we simulate a loaded cavity, the CIR is rapidly reduced and most (if not the total amount) of the energy involved in the system would be simulated.

\section{D. $R$ Coefficient}

1) Estimation: The loss coefficient used in our model is empirically determined by using a measurement of the CIR with a given loading. We used the following methodology. First, we measure

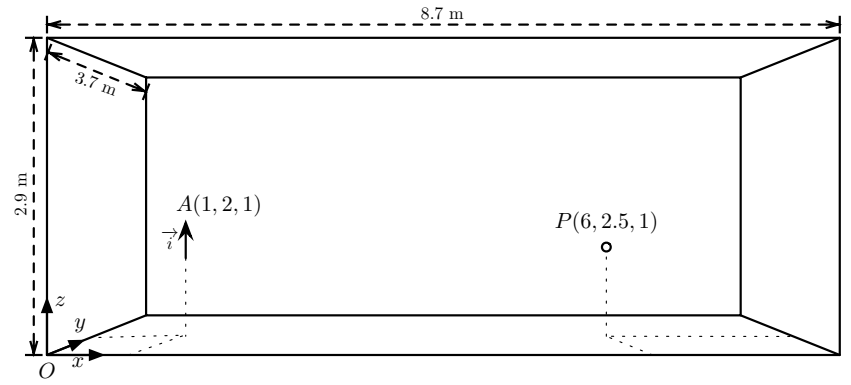

Fig. 7. Configuration of the simulated cavity.

a $\mathrm{CIR}^{8}$. We use an arbitrary waveform generator (Tektronix ${ }^{\circledR}$ AWG 7052) to create the shortest impulse possible $(200 \mathrm{ps}, 2.5 \mathrm{GHz}$ of bandwith). The antennas used are generally a pair of wide band horn antennas or discone antennas. The signal is averaged to increase the SNR and recorded on a digital storage oscilloscope (Tektronix ${ }^{\circledR}$ TDS6124C at 40 GS/s). We compute the square of the signal and we make several simulations with different values of $R$. In order to compare the simulations with the measurement, both the simulations and the measurement are normalized so that the cumulative power after $3 \mu$ s equals 1 . These simulations are compared with measurements and we identify graphically the correct value of $R$. Fig. 6 shows how the value of $R$ is chosen, the idea is to find the value of $R$ (among 501 values between 0.95 and 1) that fits the most the measurement. In this case the value $R=0.9924$ fits the measurement made in our RC with one absorber. Practically $R$ values higher than 0.995 are used to simulate an empty cavity. Values under 0.995 are used to simulate a loaded cavity. A theoretical approach to estimate $R$ and the approach evoked above are presented with more details in appendix A. As the coefficient $R$ takes in account the loss in the cavity, the relation between $R$ and the quality factor $Q$ is discussed in appendix B.

\section{Results}

\section{A. Preliminary Results}

In this section we present preliminary results of our model. We have chosen to simulate a rectangular cavity similar to the RC of our laboratory. Its dimensions are $8.7 \times 3.7 \times 2.9 \mathrm{~m}$. The emitting current is placed at the point $A$ in the left part of the cavity (Fig. 7). The results presented here are simulations conducted in a particular reception

\footnotetext{
${ }^{8}$ The effect of the electronic stirring induced by this wideband measurement implies the the CIRs measured or simulated have roughly the same profile in different locations, a single measurement is enough to estimate $R$.
} 


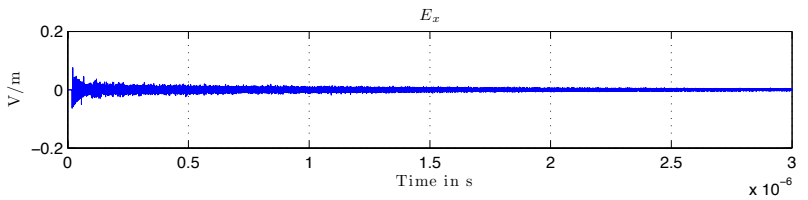

$E_{y}$

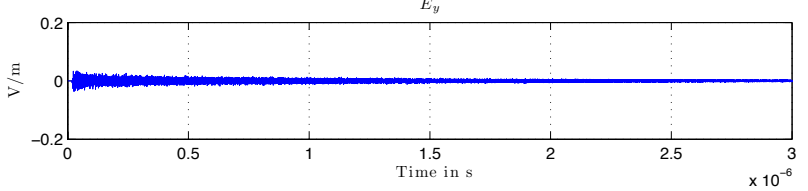

E

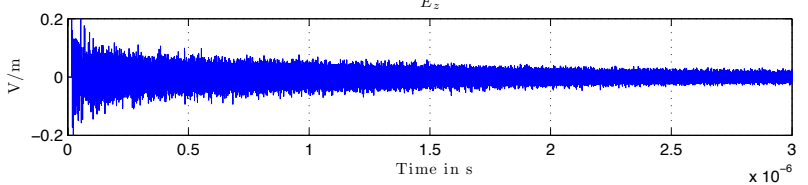

Fig. 8. Channel impulse responses (linear values) along the three rectangular components of the E-field, simulation made over $3 \mu \mathrm{s}$, with $R=R_{x}=R_{y}=R_{z}=0.995$.

point $P$ in the right part of the cavity or in a complete horizontal plane above the emitter (plane $z=2 \mathrm{~m}$ ).

Fig. 8 presents the CIR of the simulated cavity at the reception point $P$ with an emitting current oriented along the $O z$ axis. The amplitude of the signal received along the axis $O z$ is greater than the two other rectangular components. This result can be understood when we take account of the respective positions of the emitter and the receiver. Due to the very regular geometry of our cavity and the orientation of the elementary current, all the elementary image-currents are oriented along the axis $O z$ and thus the polarization along this axis is favored. The fast Fourier transforms of these CIRs (Fig. 9) show that for low frequencies, the cavity is undermoded. For frequencies lower than $200 \mathrm{MHz}$, we can clearly identify the resonant frequencies of our cavity.

Above $300 \mathrm{MHz}$ the Fourier transforms exhibit fast fading. A statistical analysis of the Fourier transforms along the three rectangular components (Fig. 10) for independent [15] frequencies between 1 and $2 \mathrm{GHz}$ shows that the rectangular components of the E-field follow Rayleigh distributions [16]. Fig. 10 shows that the component along the $O z$ axis is more scattered. As expected, the polarization at the reception is mostly the same as the emitter. Without an arbitrary-shaped stirrer, the E-field in the simulated cavity is mainly oriented along the direction of the source, in this case along the axis $O z$ (vector $(0,0,1)$ ). By changing the orientation of the elementary current along the vector $(1,1,1)$, the three rectangular components are almost equally scattered.

Fig. 11 shows the response obtained along the three rectangular components with a rectangular
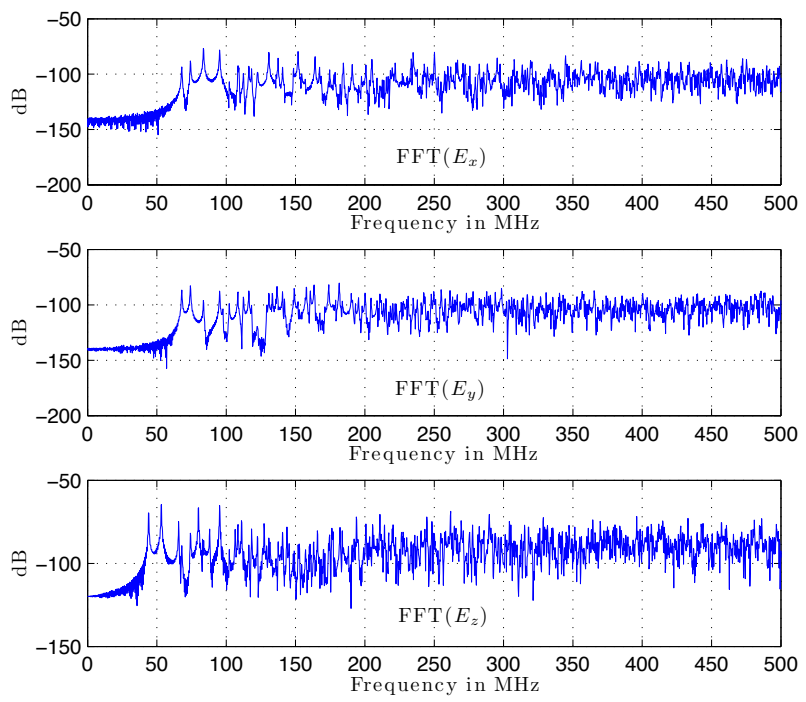

Fig. 9. Fourier transforms of the CIRs for each rectangular component (in dB).
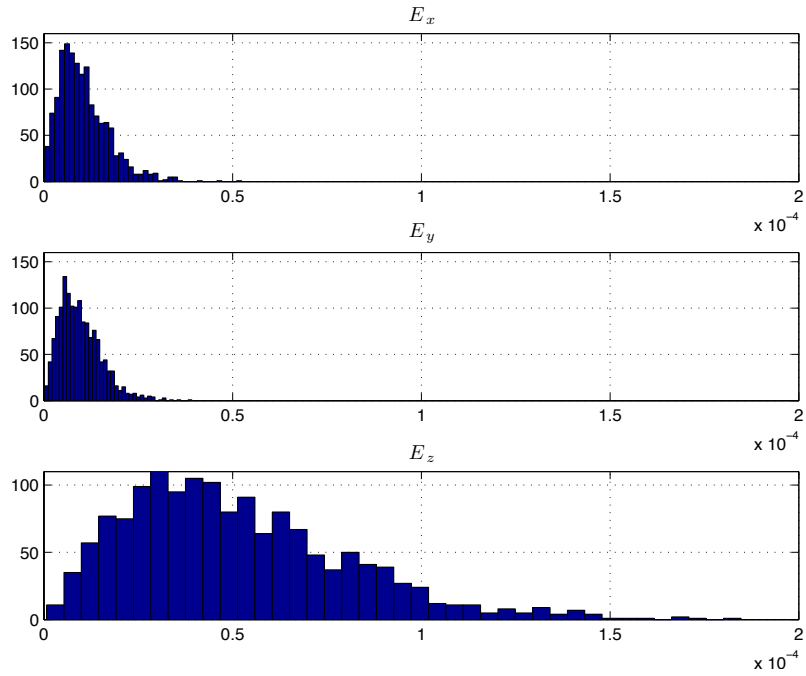

Fig. 10. Probability density function of $\operatorname{FFT}\left(E_{x}\right), \operatorname{FFT}\left(E_{y}\right)$ and $\operatorname{FFT}\left(E_{z}\right)$ over $300 \mathrm{MHz}$ around $1100 \mathrm{MHz}(1500$ samples).

monochromatic pulse signal at $1 \mathrm{GHz}$, the length of the pulse $\tau$ is $200 \mathrm{~ns}$. The complexity of the signals simulated is typical of pulse signals in a reverberation chamber.

Fig. 12 shows the propagation of the power held by a $\tau=100 \mathrm{~ns}$ long pulse at $500 \mathrm{MHz}$ in an horizontal plane of the cavity. This simulation made of 12000 receiving points shows that the emitted pulse is reflected by the walls of the cavity. We can notice that the total power in the cavity grows during the pulse emission and fades away after. The Fourier transform in an horizontal plane exhibits the characteristic cavity modes one should expect at a given frequency. Fig. 13 shows the component 

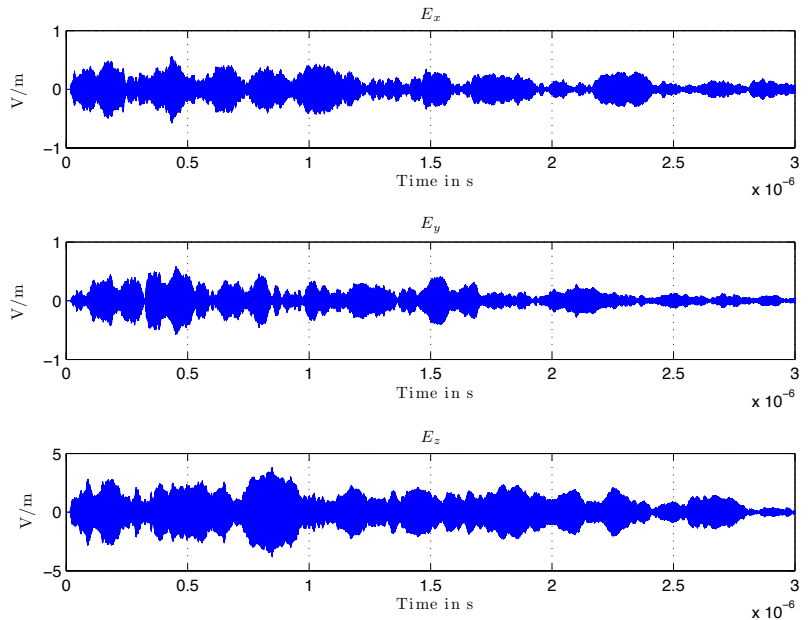

Fig. 11. E-field received (linear values) along the three rectangular components for a monochromatic pulse of $\tau=200$ ns at $1 \mathrm{GHz}$. Simulation made over $3 \mu \mathrm{s}$, with $R=R_{x}=$ $R_{y}=R_{z}=0.995$.
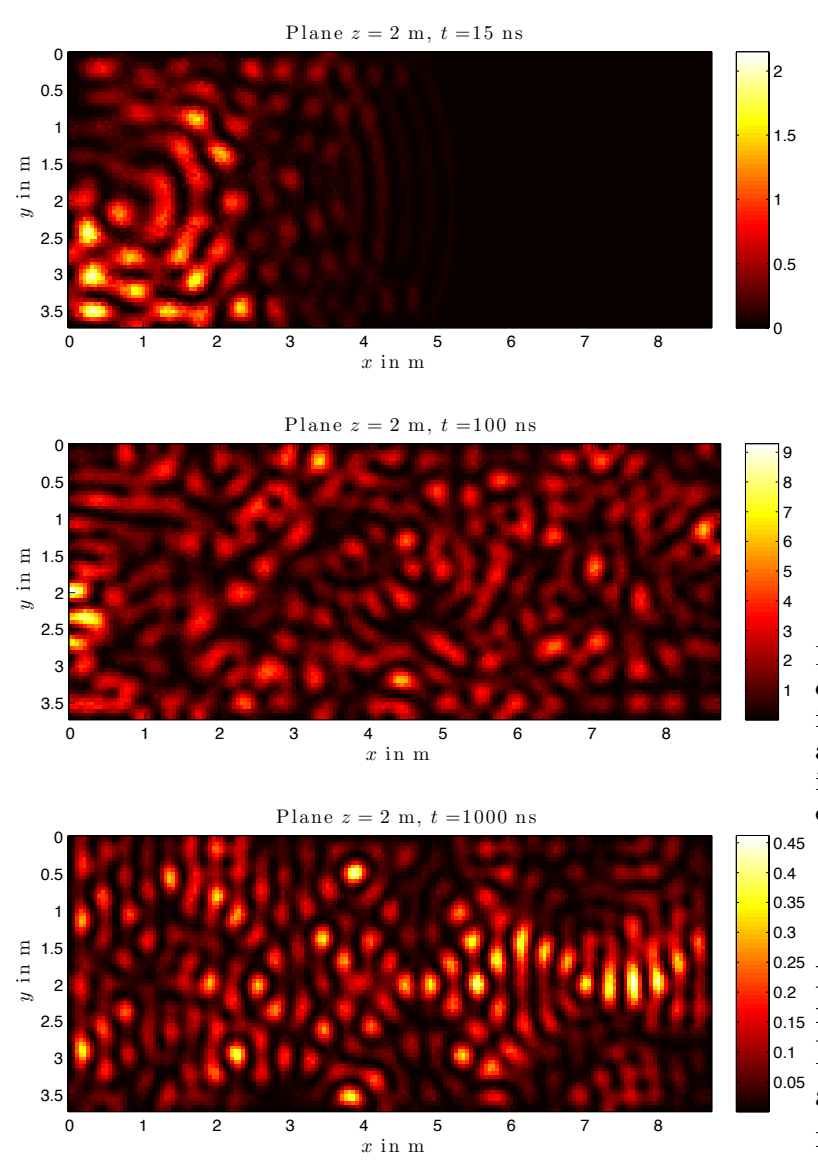

Fig. 12. Total power (linear values) in the plane $z=2 \mathrm{~m}$ for a pulse signal of length $\tau=100 \mathrm{~ns}$ at $500 \mathrm{MHz}$ at different instant $(t=15 \mathrm{~ns}, t=100 \mathrm{~ns}, t=1 \mu \mathrm{s})$. The elementary current tilt angle is $\pi / 4$ and its azimuth is $\pi / 4$. Simulation are made over $1 \mu \mathrm{s}$, with $R=R_{x}=R_{y}=R_{z}=0.995$.

along the $O z$ axis of the E-field. First, we note that the boundary conditions are respected, in
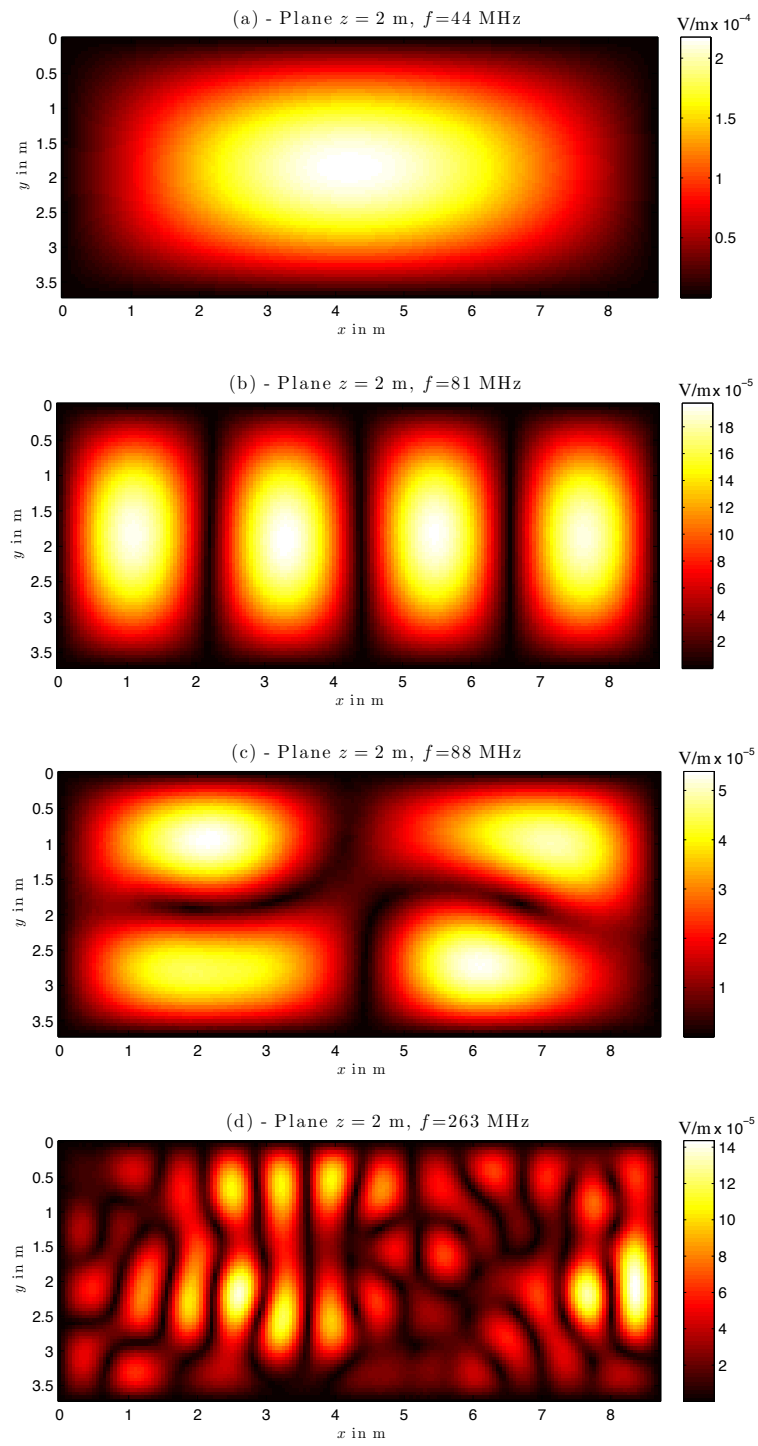

Fig. 13. Fourier transform of the $E_{z}$ component of the electric field (linear values) in the plane $z=2 \mathrm{~m}$ at different frequencies. The elementary current tilt angle is $\pi / 4$ and its azimuth is $\pi / 4$. The $\mathrm{TE}_{110}$ cavity mode (a), the $\mathrm{TE}_{410}$ cavity mode (b), the $\mathrm{TE}_{220}$ cavity mode (c), and a combination of different cavity modes around the LUF (d) are presented.

particular, the $E_{z}$ component of the E-Field is null along the walls. The first three pictures in Fig. 13 show typical cavity modes. We have been able to identify all the modes by using the resonant frequencies formula for a rectangular cavity [10] with $l=8.7 \mathrm{~m}, p=3.7 \mathrm{~m}$, and $h=2.9 \mathrm{~m}$ :

$f_{x y z}=\frac{c}{2} \sqrt{\left(\frac{x}{l}\right)^{2}+\left(\frac{y}{p}\right)^{2}+\left(\frac{z}{h}\right)^{2}}$, with $x, y, z \in \mathbb{N}$.

The bottom picture in Fig. 13 is around $260 \mathrm{MHz}$ (around the LUF of our cavity), different cavity modes are combined and the distribution of the power is not regular.

These preliminary results are consistent with ob- 


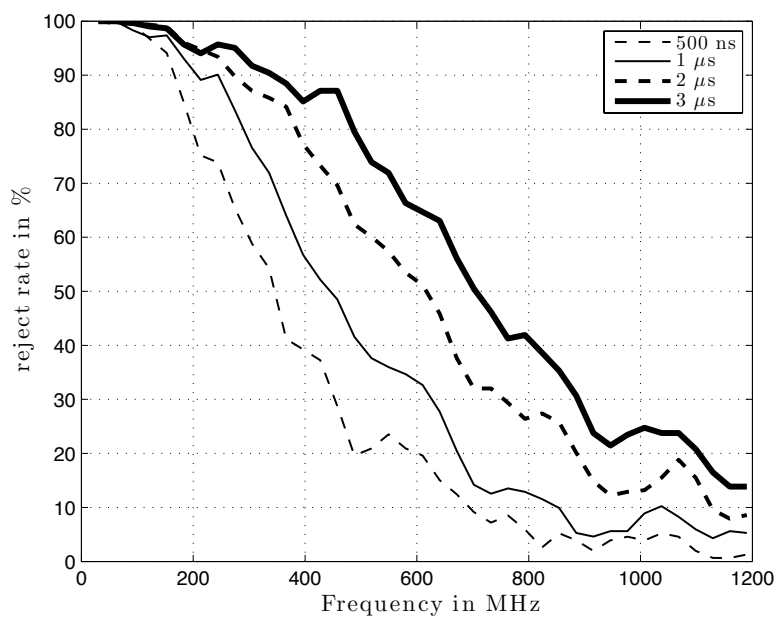

Fig. 14. Reject rate with the AD GoF test for the Rayleigh distribution for rectangular components of the E-field with $N=150$ for different lengths of CIR, and an empty cavity $(R=0.998)$.

servations made in a real $\mathrm{RC}$ and with rectangular cavity theory.

\section{B. Statistical Frequency Domain Exploration}

In this section, we simulate the chamber of our laboratory without loading. Usually the calibration of an RC is done without loading. By choosing to simulate the case of an empty cavity, we choose the worst case scenario in terms of convergence of the CIR and we will be able to compare the simulations with measurements that have been made during the past years. As presented in Section II-C2, we cannot simulate a full CIR if the cavity is not loaded. To perform a study of the frequency domain, we use Fourier transforms of truncated CIRs. The length of the simulated CIR affects directly the frequency response. When the frequency response is very short, the fast Fourier transform of the CIR widens the resonances. When the CIR is longer the widening is less pronounced. This widening is more important at lower frequencies. As a result, the modes are artificially combined at low frequencies as if the quality factor $Q$ is relatively low. In order to study the statistic properties of the rectangular components of the E-field, we use the Anderson-Darling (AD) goodness of fit test (GoF). First we calculate $N$ CIRs in the cavity by moving the receiver. A fast Fourier transform is applied to these CIRs giving a $N$ size sample for every independent frequency [15]. Each sample at a given frequency is tested with the $\mathrm{AD}$ GoF test for Rayleigh distribution with Stephen's values [17]. The null hypothesis $H_{0}$ is accepted when the population composed of the $N$ observations follows a Rayleigh distribution. On the contrary the alternative hypothesis $H_{1}$ favors a distribution that

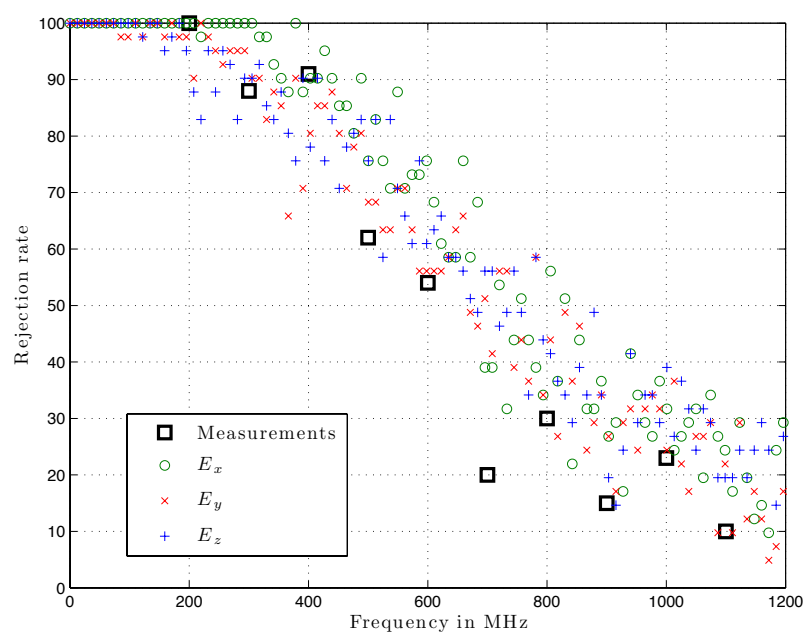

Fig. 15. Rejection rate in \% with Anderson-Darling goodness of fit test for the Rayleigh distribution with $N=100$. Results from E-field measurements (along a rectangular component) and from simulated E-field (three rectangular components) with $R=R_{x}=R_{y}=R_{z}=0.998$.

is not Rayleigh distributed. The level of significance $\alpha=0.05$ means that the test will fail to recognize $5 \%$ percent of the Rayleigh distributions. Figure 14 shows the effect of the length of the CIR on the statistics obtained for the rectangular components of the E-field. We can notice that the Rayleigh distribution is largely accepted after $400 \mathrm{MHz}$ when the CIR is only 500 ns long. Measurements have shown that the Rayleigh distribution is largely accepted only after $900 \mathrm{MHz}$ with these tests. With 2 or $3 \mu$ s long CIRs, the simulations fit the measurements (fig. 15). The results obtained with our model are compared with results extracted from measurements made in our RC [17]. These measurements were performed with an isotropic three-axis field probe (Hi6005) and amplifiers. The field was measured for 30 independent positions of probe and at least 30 independent positions of stirrer. The samples are then grouped in series of $N=100$ independent samples [17]. Fig. 15 shows the rejection rate of the $\mathrm{AD}$ GoF statistical test for different frequencies for a measured rectangular component of the E-field and for three rectangular components simulated in an empty cavity. For frequencies under $300 \mathrm{MHz}$, the null hypothesis $H_{0}$ is massively rejected. This hypothesis is more and more accepted when the frequency increases. The rejection rates from the measurements and our simulations for the considered frequency range are very similar. These results are encouraging, globally it seems that the frequency domain behavior of our simulated cavity fits the behavior of our real RC even if we cannot simulate a full CIR. 


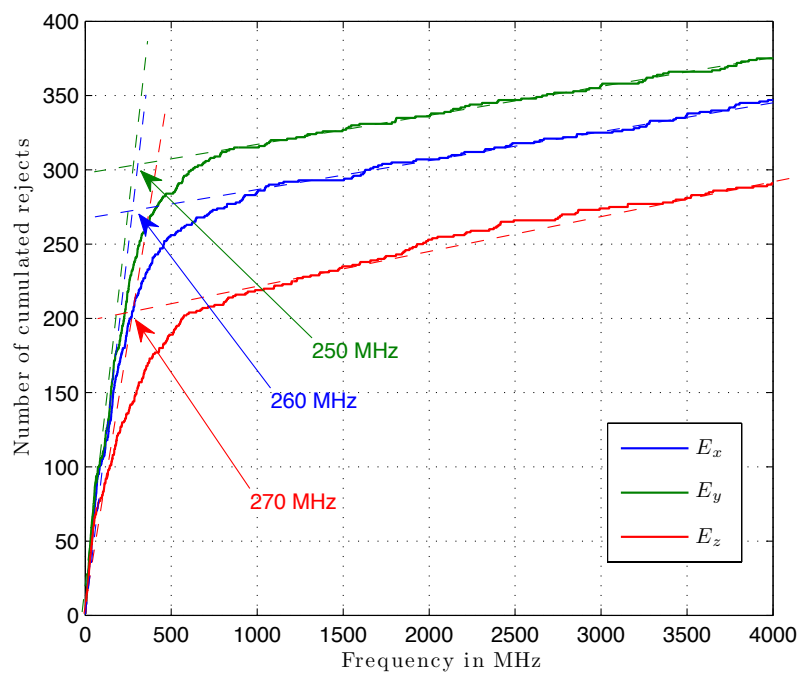

Fig. 16. Cumulated number of rejected tests for the three rectangular components of the E-field. The elementary current tilt angle is $\pi / 4$ and its azimuth is $\pi / 4$.

\section{C. $L U F$}

The LUF is a characteristic constant of an $\mathrm{RC}$ [18]. Above the LUF, the modes involved in the cavity are combined and the fields in the cavity are stochastic. In particular, the components of the electric field follow a Rayleigh distribution [16]. We developed an original approach to determine the LUF with this model. It consists in calculating $N$ CIRs in the cavity by moving the receiver. A fast Fourier transform is applied to these CIRs giving a $N$ size sample for every independent frequency. [15]. Each sample is tested with the Anderson Darling statistical test [17] with Stephen's values. The level of significance chosen is $\alpha=0.01$, it means that the test is very severe and thus the graphical analysis of the results will be easier.

The results for a cavity matching the dimensions of the RC in our laboratory are presented in Fig. 16. The functions of the cumulative number of rejected tests for each rectangular component exhibit the same tendencies. Under the LUF, the null hypothesis is massively rejected and the slope is important. Around $200 \mathrm{MHz}$, the null hypothesis is more accepted and the slope of the cumulative number of rejects decreases. Above $500 \mathrm{MHz}$ the null hypothesis is largely accepted and the slope equals $\alpha$. These three distinct trends were noticed in [17]. If we trace the two asymptotes, for low frequencies and for high frequencies, they intersect around the LUF. The LUF of our RC is around 250 $\mathrm{MHz}$. The results presented in Fig. 16 show that the LUF given by our model is around $260 \mathrm{MHz}$ and is in agreement with the value commonly used in our chamber.

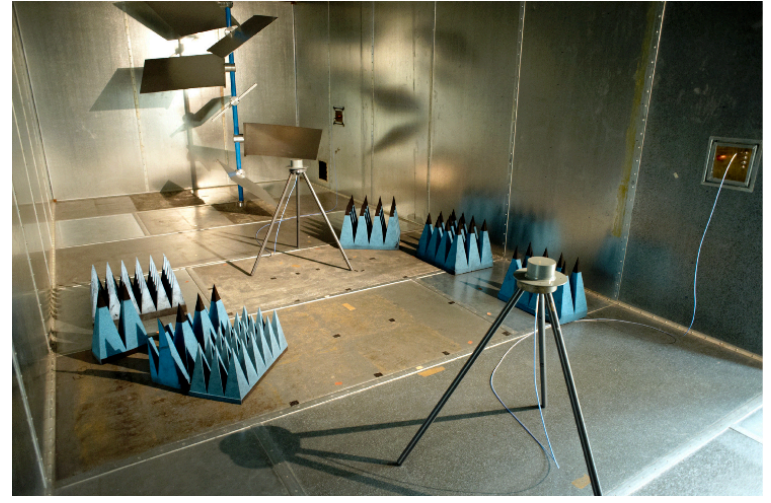

Fig. 17. Experimental configuration.

\section{Levels for Immunity Testing in the Pulsed Mode}

To conduct immunity testing, one should know the mean and the maximum levels a device under test (DUT) will receive during the test [18]. We want to know if our model is able to predict the levels and the average waveforms for a given loading and a given signal. Our RC is loaded with absorbers (Fig. 17) and the emitting antenna and the DUT are both wide-band discone antennas. The first step is to measure an impulse channel response. The duration of the CIR gives an estimation of the loss coefficients used in the model. We found $R=R_{x}=R_{y}=R_{z}=0.98$. Then the pulse signal $(\tau=300 \mathrm{~ns}$ at $1 \mathrm{GHz})$ is emitted. The receiving antenna is moved in the $\mathrm{RC}$. We made 50 measurements in our RC. The situation is reproduced in the model, using 50 simulations in 50 arbitrary positions in our virtual cavity. From both the measurements and the simulations, we extract the envelopes of the signals and we compute the average power of the levels received and the maximum power for every time-step. The levels obtained by simulations in $\mathrm{dB}$ are corrected by adding a constant bias to fit the measurements of the mean and the max. Fig. 18(a) shows a good agreement between the measurements and the simulations. Fig. 18(b) shows that the ratio $\max /$ mean is around $6.5 \mathrm{~dB}$ in both the simulations and the measurements. This value is characteristic of an observation over $N=50$ [19], [20]. In linear values, Fig. 19, we can note that measured and simulated averages of the power are very similar. Our model simulates with a good accuracy the waveforms obtained in the pulse regime.

\section{COnClusion}

\section{A. Main Results}

With very simple assumptions and straightforward calculations, the model we propose is able to statistically reproduce the behavior of an RC. Frequency domain simulations show properties similar 

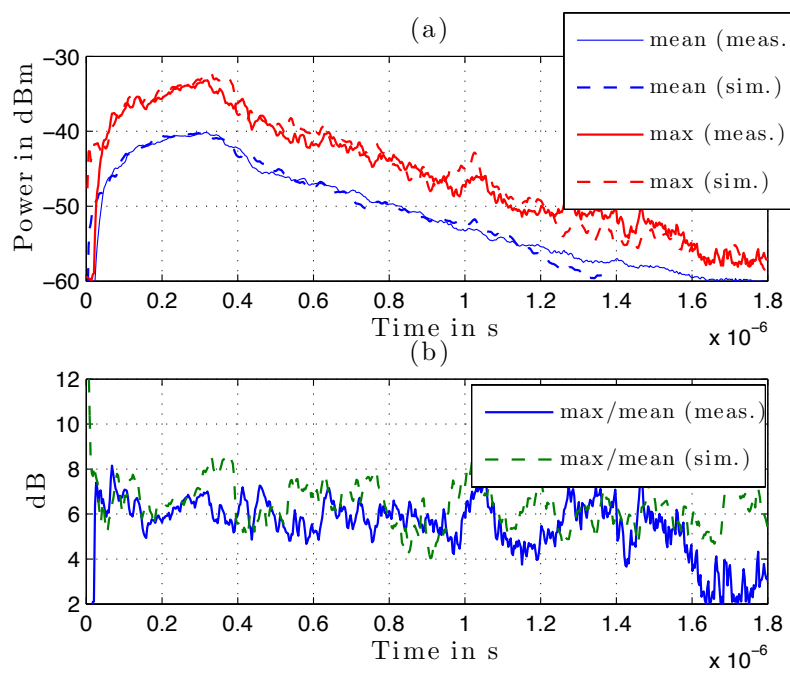

Fig. 18. (a) Maximum and mean power (in dBm) from measurements and simulations. Maximum/mean ratio (in $\mathrm{dB}$ ) from measurements and simulations for a $300 \mathrm{~ns}$ long pulse at $1 \mathrm{GHz}$.

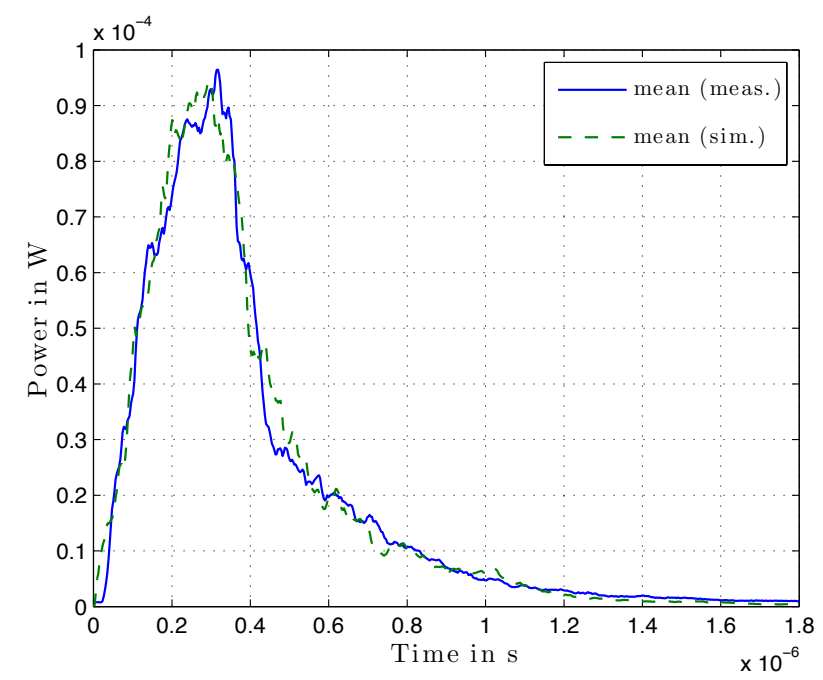

Fig. 19. Normalized mean power from measurements and simulations for a $300 \mathrm{~ns}$ long pulse at $1 \mathrm{GHz}$.

to a real RC. Time domain simulations exhibit similar behavior and levels. This model is able to explore both the frequency domain and the time domain. It can be helpful to conduct immunity testing in the pulse regime in an $\mathrm{RC}$ as well as rapidly study the influence of various parameters (dimensions, loading, directivity) in the frequency domain.

\section{B. General Discussion and Modifications Needed}

It may be advantageous to use this model to extract general considerations about the effect of the loading on the performance of the $\mathrm{RC}$ and about the transients. For instance, it may be helpful to understand how the RC stores the energy emitted by an antenna and how the directivity of the emitting source vanishes. This model based on CIRs and convolutions can also be helpful to simulate rapidly time-reversal experiments.

Further developments of the model are to be considered. We are currently trying to add a stirring method to our model in order to add a degree of freedom to our simulations and to apply the battery of statistical methods developed in the past years to compare our model to real RCs measurements. We are currently working on a stirring process. Our first approach would be to simulate an amount of $M$ situations, in which the position of the emitting elementary dipole is different. By applying the superposition theorem, a stirring step could be a combination of $N$ simulations among $M$. Another approach could be to change the dipole radiation pattern by a more directive pattern and to simulate a paddle with five or six of these directive sources rotating around a chosen axis.

\section{ApPENDix A}

\section{Estimation of the LOSS COEFFICIENT $R$}

In the following, we assume that the CIR envelope is an exponential function. The rule of conservation of energy dictates the amount of power radiated by every image current in the system. At the order $i$, the $\left(4 i^{2}+2\right)$ cavities are approximately placed on a sphere of radius $i d$, where d is a dimension of the reverberation chamber. The fraction of power emitted by these sources that would reach the initial cavity after a time delay $t=i d / c$ is approximately $\left(4 \pi /\left(4 i^{2}+2\right)\right)$. We can approximate the incoming simulated E-field from the $\left(4 i^{2}+2\right)$

cavities of order $i$ in the initial cavity by:

$$
\left|E_{s}(i)\right| \approx E_{s_{0}} R^{i} \approx E_{s_{0}} R^{t \frac{c}{d}} \approx E_{s_{0}} e^{t \frac{c}{d} \ln R}
$$

We can express the envelope of the measured Efield with an exponential profile:

$$
\left|E_{m}(t)\right|=E_{m_{0}} e^{-t / \tau},
$$

With (14)and (15) we can approximate a relation between the "time to live" parameter $\tau$ and $R$. If the dimensions of the rectangular cavity considered are $l, p, h$ and that $h$ is the smallest dimension, the time between two reflections is at least $h / c$. Therefore:

$$
\tau \approx-\frac{h}{c \ln R}
$$

and because $R=1-\epsilon$ with $\epsilon \ll 1$,

$$
\tau \approx-\frac{h}{c \ln (1-\epsilon)} \approx \frac{h}{c \epsilon} \approx \frac{h}{c(1-R)} .
$$

We can deduce an approximative value of $R$ :

$$
R \approx 1-\frac{h}{c \tau} \text {. }
$$


From the measurements, we find $\tau=6 \mu$ s when the cavity is empty. If we apply this formula with $h=2.9 \mathrm{~m}$, we find $R \approx 0.998$. This rough approach gives good results.

The method we used to determine $R$ consists in finding the $R$ value that minimizes the error between simulations and measurements. We generate a great amount of simulations with $R$ varying from 0.95 to 1 . By computing the power delay profile from a CIR measurement, we have:

$$
P_{m}(t)=P_{m_{0}} e^{-2 t / \tau},
$$

the energy measured $\mathcal{E}_{m}(t)$ received till $t$ is given by:

$$
\mathcal{E}_{m}(t)=\int_{0}^{t} P_{m}(t) d t=\frac{1}{2} P_{m_{0}} \tau\left(1-e^{-2 t / \tau}\right) .
$$

The energy from the simulations is given by:

$$
\mathcal{E}_{s}(t)=\int_{0}^{t} P_{s}(t) d t=\frac{1}{2} P_{s_{0}} \tau_{s}(R)\left(1-e^{-2 t / \tau_{s}(R)}\right),
$$

where $\tau_{s}(R)$ is the "time to live" parameter in our simulations as a function of the loss coefficient $R$. The energy functions are scaled after $t_{0}=3 \mu \mathrm{s}$ because the levels of the simulations with different values of $R$ are different. We obtain:

$$
\mathcal{E}_{m}\left(t_{0}\right)=A \mathcal{E}_{s}\left(t_{0}\right)
$$

where $\mathrm{A}$ is the scaling factor.

And we try to find the value of $\mathrm{R}$ that minimizes the error Err:

$$
\operatorname{Err}=\int_{0}^{t_{0}}\left|\mathcal{E}_{m}(t)-A \mathcal{E}_{s}(t)\right| d t .
$$

Both methods are equivalent. The problem with the first method is that we need to express the number of reflections in unit of time. With our chamber it works well if we consider the smallest dimension. But our chamber is very long $(8.7 \times$ $2.7 \times 2.9 \mathrm{~m})$ and we can easily understand that the reflections along the height of our chamber are more numerous than the two other dimensions and so are mainly responsible for the power decay observed. The second method that involves numerous simulations of a particular chamber, takes in account the dimensions of the chamber and may be more accurate.

\section{APPENDIX B}

\section{Discussion ON THE RELATION BETWEEN $R$ AND} $Q$

The purpose of this appendix is to establish a link between the quantity $R$ defined in this paper and the quality factor $Q$. Theoretical expressions of the quality factor of an $\mathrm{RC}$ integrate the conductivity of the materials used in an $\mathrm{RC}$ and the dimensions of the considered RC [21], [22]. The quality

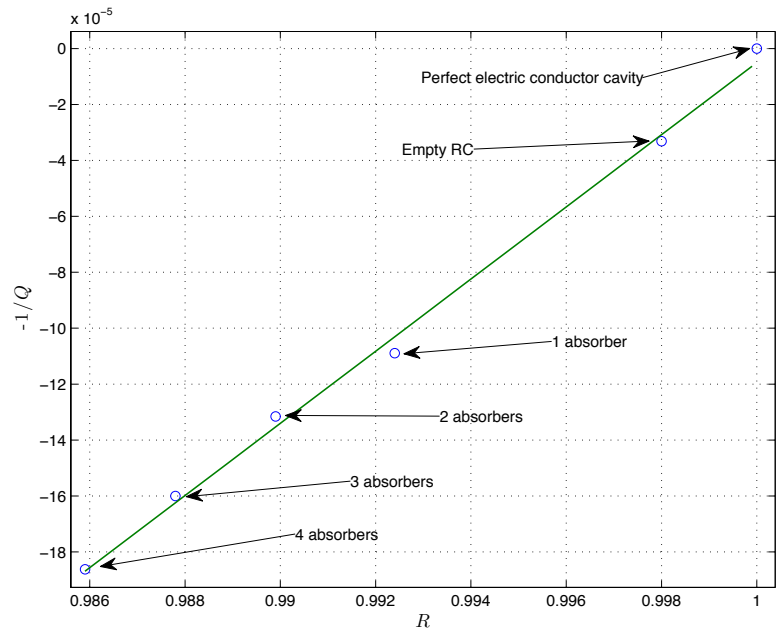

Fig. 20. Quantity $-1 / Q$ vs. $R$ for different loading and linear fitting. The slope $\mathcal{A} \approx 0.013$.

factor extracted from measurements includes the losses from the cavity and every object inside. As our loss coefficient $R$ integrates all the losses of our system, we can certainly derive a relation between $R$ and $Q$. Measurements of the quality factor of our RC were conducted from $200 \mathrm{MHz}$ to $2 \mathrm{GHz}$. CIR measurements were done with a $200 \mathrm{ps}$ long impulsion giving a $2.5 \mathrm{GHz}$ bandwidth. The antennas used were two identical wide band horn antennas $(1-18 \mathrm{GHz})$. The values of the quality factor $Q$ used here are an averaging of $Q(f)$ with $f$ from $1 \mathrm{GHz}$ to $2 \mathrm{GHz}$. We used four identical pieces of rectangular absorbers. Fig. 20 shows that there must be a linear relation between the quantities $R$ and $-1 / Q$ :

$$
R \approx 1-\frac{1}{\mathcal{A} Q}
$$

We found $\mathcal{A} \approx 0.013$. This value of $\mathcal{A}$ found empirically is relevant for our cavity only. Values of $\mathcal{A}$ may vary with the dimensions of the cavity, the conductivity of the walls, and the various lossy elements found in an RC.

It can be interesting to find an approximative expression of $\mathcal{A}$. If we assume the exponential profile of the CIR, the quality factor $Q$ at the frequency $f_{0}$ is given by the time domain expression of the quality factor $Q=2 \pi f_{0} \tau$ [23], if we consider (18) and (24), we can write:

$$
\begin{aligned}
\frac{h}{c \tau} & \approx \frac{1}{2 \pi \mathcal{A} f_{0} \tau}, \\
\mathcal{A} & \approx \frac{c}{2 \pi f_{0} h} .
\end{aligned}
$$

With $h=2.9 \mathrm{~m}$ and $f_{0}$ between 1 and $2 \mathrm{GHz}$, the average value of $\mathcal{A}$ obtained is 0.011 which is very similar to the value obtained with the measurements of the quality factor in our cavity. 


\section{ACKNOWLEDGMENTS}

This work was supported by the French Ministry of Defence DGA (Délégation Générale de l'Armement), with a Ph.D. grant delivered to Emmanuel Amador and a"REI" grant 200834004 delivered to IETR (INSA de Rennes). The authors would like to thank Jérôme Sol for his assistance during the measurements.

\section{REFERENCES}

[1] O. Lunden and M. Backstrom, "Pulsed power $3 \mathrm{GHz}$ feasibility study for a $36.7 \mathrm{~m}^{3}$ mode stirred reverberation chamber feasibility study for a $36.7 \mathrm{~m}^{3}$ mode stirred reverberation chamber," in Electromagnetic Compatibility, 200\%. EMC 200\%. IEEE International Symposium on, July 2007, pp. 1-6.

[2] - "Absorber loading study in foi $36.7 \mathrm{~m}^{3}$ mode stirred reverberation chamber for pulsed power measurements," in Electromagnetic Compatibility, 2008. EMC 2008. IEEE International Symposium on, Aug. 2008, pp. 1-5.

[3] L. Arnaut, "Time-domain measurement and analysis of mechanical step transitions in mode-tuned reverberation: Characterization of instantaneous field," Electromagnetic Compatibility, IEEE Transactions on, vol. 49, no. 4, pp. 772-784, Nov. 2007.

[4] C. Bruns and R. Vahldieck, "A closer look at reverberation chambers - 3-D simulation and experimental verification," Electromagnetic Compatibility, IEEE Transactions on, vol. 47, no. 3, pp. 612-626, Aug. 2005.

[5] M. Höijer, A.-M. Andersson, O. Lunden, and M. Backstrom, "Numerical simulations as a tool for optimizing the geometrical design of reverberation chambers," in Electromagnetic Compatibility, 2000. IEEE International Symposium on, vol. 1, 2000, pp. 1-6.

[6] A. Coates, H. Sasse, D. Coleby, A. Duffy, and A. Orlandi, "Validation of a three-dimensional transmission line matrix (TLM) model implementation of a modestirred reverberation chamber," Electromagnetic Compatibility, IEEE Transactions on, vol. 49, no. 4, pp. 734-744, Nov. 2007

[7] G. Orjubin, F. Petit, E. Richalot, S. Mengue, and O. Picon, "Cavity losses modeling using lossless FDTD method," Electromagnetic Compatibility, IEEE Transactions on, vol. 48, no. 2, pp. 429-431, May 2006.

[8] D.-H. Kwon, R. Burkholder, and P. Pathak, "Ray analysis of electromagnetic field build-up and quality factor of electrically large shielded enclosures," Electromagnetic Compatibility, IEEE Transactions on, vol. 40, no. 1, pp. 19-26, Feb 1998.

[9] S. Baranowski, D. Lecointe, M. Cauterman, and B. Demoulin, "Use of $2 \mathrm{D}$ models to characterize some features of a mode stirred reverberation chamber," vol 9-13. International Symposium on Electromagnetic Compatibility, Sorrento, Italy, september 2002, pp. 381-386.

[10] R. Harrington, Time-Harmonic Electromagnetic Fields. New York: McGraw-Hill Book Company, 1961, pp. 103-105.

[11] Y. Huang and D. Edwards, "A novel reverberating chamber: the source-stirred chamber," Sep 1992, pp $120-124$.

[12] C. Monteverde, G. Koepke, C. Holloway, J. Ladbury, D. Hill, V. Primiani, and P. Russo, "Source stirring technique for reverberation chambers; experimental investigation," Sept. 2008, pp. $1-6$.

[13] G. Cerri, V. Primiani, C. Monteverde, and P. Russo, "A theoretical feasibility study of a source stirring reverberation chamber," Electromagnetic Compatibility, IEEE Transactions on, vol. 51, no. 1, pp. $3-11$, Feb. 2009.
[14] J. Kunthong and C. Bunting, "Statistical characterization of the $900 \mathrm{MHz}$ and $1800 \mathrm{MHz}$ indoor propagation using reverberation source stirring technique," in $A n$ tennas and Propagation Society International Symposium, 2009. APSURSI '09. IEEE, June 2009, pp. 1-4.

[15] C. Lemoine, P. Besnier, and M. Drissi, "Estimating the effective sample size to select independent measurements in a reverberation chamber," Electromagnetic Compatibility, IEEE Transactions on, vol. 50, no. 2, pp. 227-236, May 2008.

[16] D. Hill, "Plane wave integral representation for fields in reverberation chambers," IEEE Transactions on Electromagnetic Compatibility, vol. 40, no. 3, pp. 209-217, Aug 1998.

[17] C. Lemoine, P. Besnier, and M. Drissi, "Investigation of reverberation chamber measurements through highpower goodness-of-fit tests," Electromagnetic Compatibility, IEEE Transactions on, vol. 49, no. 4, pp. 745755, Nov. 2007.

[18] "IEC 61000-4-21: Electromagnetic compatibility (EMC) - part 4-21: Testing and measurement techniques - reverberation chamber test methods," IEC, Tech. Rep., 2003.

[19] J. Ladbury, G. Koepke, and D. Camell, "Evaluation of the NASA Langley research center mode-stirred chamber facility," NIST, Technical Note 1508, January 1999.

[20] M. Höijer, "Maximum power available to stress onto the critical component in the equipment under test when performing a radiated susceptibility test in the reverberation chamber," Electromagnetic Compatibility, IEEE Transactions on, vol. 48 , no. 2, pp. $372-384$, May 2006.

[21] D. Hill, "A reflection coefficient derivation for the Q of a reverberation chamber," Electromagnetic Compatibility, IEEE Transactions on, vol. 38, no. 4, pp. 591-592, Nov 1996.

[22] P. Corona, G. Ferrara, and M. Migliaccio, "A spectral approach for the determination of the reverberating chamber quality factor," Electromagnetic Compatibility, IEEE Transactions on, vol. 40, no. 2, pp. 145-153, May 1998.

[23] N. Hodgson and H. Weber, Optical Resonators, Fundamentals, Advanced Concepts and Applications, 1st ed. London, Great Britain: Springer-Verlag, 1997, p. 142.

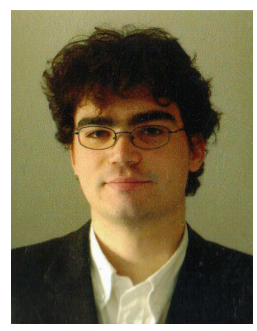

Emmanuel Amador (S'10) received the Diplôme d'Ingénieur degree from Institut National des Télécommunications (Télécom INT), Evry, France in 2006. He received his M.Sc. in electrical engineering from Laval University, Quebec City, QC, Canada in 2008. He is currently a Ph.D. student in electronics at the Institute of Electronics and Telecommunications of Rennes (IETR), INSA of Rennes, France. 


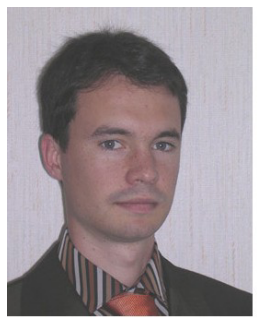

Christophe Lemoine received the Diplôme d'Ingénieur degree from Ecole Nationale Supérieure de l'Aéronautique et de l'Espace (SUPAERO), Toulouse, France, in 2004. He received a Master degree in financial risk management in 2005, and then was pursuing the Ph.D. degree in electronics from INSA of Rennes, France (defended in 2008). His current research interest at the Institute of Electronics and Telecommunications of Rennes (IETR), Rennes, France, includes new theoretical and experimental approaches of mode-stirred reverberation chambers for EMC, propagation channels and antenna measurement applications.

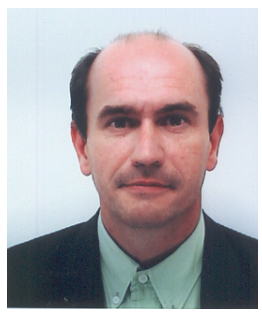

Philippe Besnier (M'04) received the diplôme d'ingénieur degree from Ecole Universitaire d'Ingénieurs de Lille (EUDIL), Lille, France, in 1990 and the Ph.D. degree in electronics from the university of Lille in 1993. Following a one year period at ONERA, Meudon as an assistant scientist in the EMC division, he was with the Laboratory of Radio Propagation and Electronics, University of Lille, as a researcher at the Centre National de la Recherche Scientifique (CNRS) from 1994 to 1997. From 1997 to 2002, he was the Director of Centre d'Etudes et de Recherches en Protection Electromagnétique (CERPEM) : a non-profit organization for research, expertise and training in EMC, and related activities, based in Laval, France. He co-founded TEKCEM in 1998, a private company specialized in turn key systems for EMC measurements. Since 2002, he has been with the Institute of Electronics and Telecommunications of Rennes, Rennes, France, where he is currently a researcher at CNRS heading EMC-related activities such as EMC modeling, electromagnetic topology, reverberation chambers, and near-field probing.

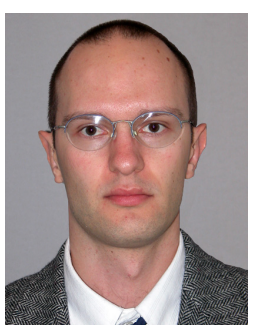

Alexandre Laisné Alexandre Laisné was born in Villedieules-pôeles, France, in 1976. He received the Master of Electrical and Electronic Engineering from Strathclyde University, Glasgow, in 1999, as well as the Electrical Engineering diploma and the Ph.D. in Electronics from the National Institute of Applied Sciences of Rennes, respectively in 1999 and 2002. After research works at Rutherford Appleton Laboratory, United Kingdom, in 2002 and the Institute of Electronics and Telecommunications of Rennes in 2003, he joined the Centre d'Essais Aéronautique de Toulouse (Direction Générale de l'Armement) in 2004. He is currently Head of the Numerical Simulation Department. His research interests include numerical simulation, EMC, Reverberation Chambers, High Intensity Radiated Fields, lightning, SAR and Hazards of Electromagnetic Radiation to Ordnance. 